\title{
Synthesis of auxetic structures using optimization of compliant mechanisms and a micropolar material model
}

\author{
Matteo Bruggi · Valentina Zega - Alberto Corigliano
}

Received: date / Accepted: date

\begin{abstract}
Aim of this work is the synthesis of auxetic structures using a topology optimization approach for micropolar (or Cosserat) materials. A distributed compliant mechanism design problem is formulated, adopting a SIMP-like model to approximate the constitutive parameters of $2 \mathrm{D}$ micropolar bodies. The robustness of the proposed approach is assessed through numerical examples concerning the optimal design of structures that can expand perpendicularly to an applied tensile stress. The influence of the material characteristic length on the optimal layouts is investigated. Depending on the inherent flexural stiffness of micropolar solids, truss-like solutions typical of Cauchy solids are replaced by curved beam-like material distributions. No homogenization technique is implemented, since the proposed design approach applies to elements made of microstructured material with prescribed properties and not to the material itself.
\end{abstract}

Keywords topology optimization - Cosserat solids . auxetic structures · compliant mechanisms

Matteo Bruggi

E-mail: matteo.bruggi@polimi.it

Valentina Zega

E-mail: valentina.zega@polimi.it

Alberto Corigliano

E-mail: alberto.corigliano@polimi.it

Department of Civil and Environmental Engineering Politecnico di Milano

P.zza Leonardo da Vinci, 32 I20133, Milano, Italy

\section{Introduction}

The classical Cauchy continuum model suffers from limitations when addressing the mechanical behavior of materials endowed with a microstructure. This is the case of cellular, biological, fiber reinforced and granular materials, for instance. A more sophisticated theory than Cauchy's one is needed to describe solids where the stress gradients involve regions of a size comparable with that of the microstructure itself.

A renewed interest in the Cosserat continuum theory (see e.g. Eringen 1966) is explained by the need of investigating not only micro-structured materials (Anderson \& Lakes 1994; Yuan \& Tomita 2001; Bigoni \& Drugan 2006; Li et al. 2010), but also poly-crystalline metals (Forest et al. 2000) and poly-silicon thin films. The latter exhibit a micro-structure that is mainly tied to the grain size. In general, this measure is not negligible with respect to the magnitude of many parts of Micro Electro-Mechanical System (MEMS), see in particular Corigliano et al. (2004), Mariani et al. (2011) and Koskinent et al. (1993).

A size effect is predicted in the bending of plates and beams and in the torsion of cylinders made of Cosserat elastic materials. Slender elements appear more stiff than predicted through the classical elasticity theory. Also, small holes exhibit less stress peaks than larger ones, providing enhanced toughness to micropolar solids. The micropolar (or Cosserat) continuum model resorts to the adoption of one or more internal lengths, the socalled characteristic lengths of the material, which allow taking size effects into account. These material parameters can be derived either experimentally (Lakes 1995) or numerically through homogenization theory (Forest \& Sab 1998; Fatemi et al. 2003). 
Numerical investigations have been performed to assess the effect of the microstructure on the optimal design of structures. Topology optimization is a mathematical tool that searches for the distribution of linear elastic isotropic material to minimize a prescribed objective function for an assigned set of constraints, see e.g. Bendsøe \& Sigmund (2003) and Deaton \& Grandhi (2014). A conventional formulation minimizes the socalled structural compliance to achieve the stiffest layout using a limited amount of material (Bendsøe \& Kikuchi 1988). Rovati \& Veber (2007), Liu \& Su (2010) and Veber \& Taliercio (2011) firstly embedded the Cosserat continuum model in this formulation showing that trusslike layouts can be replaced by bending-resistant layouts depending on the value of the characteristic length. This was later confirmed by Bruggi \& Taliercio (2012) and Su \& Liu (2015), dealing with a min-max problem involving the natural frequencies of micropolar bodies.

Topology optimization has been successfully adopted to perform material design, see in particular the synthesis of extremal composites with negative Poisson's ratio (Sigmund 1994) and prescribed elasticity tensor (Sigmund 2000), or the achievement of materials with assigned thermal and electro-thermal properties (Sigmund \& Torquato 1999). The application of topology optimization involving inverse homogenization is an active field of research. Recent examples include the works by Coelho et al. (2011), who implemented a multi-scale formulation to design a trabecular bone section, and by Diaz \& Sigmund (2010), who performed the optimization of a meta-material looking for negative electromagnetic permeability. Extensive applications of microstructure design through topology optimization can be found e.g. in Cadman et al. (2012) and Andreassen et al. (2014).

Instead of resorting to homogenization theory and material design, this contribution focuses on the topology optimization of auxetic structures (and micro- structures) through a formulation for the synthesis of distributed compliant mechanisms made of micropolar material.

The most popular feature of auxetic structures is that they expand in the direction perpendicular to an externally exerted tension. This property makes auxetic structures strongly appealing for MEMS applications (i.e. motion conversion and resonators) (Levy et al. 2006). Also, auxetic materials are of interest because of the enhanced properties related to their negative Poisson's ratio, such as increased shear modulus, indentation resistance, fracture toughness, energy absorption, porosity/permeability variation with strain and synclastic curvature. Due to the scale-independence of the theory of elasticity, the auxetic behavior does not depend on the scale: deformation can take place at the nano- (molecular), (Yang et al. 2004), micro- (Larsen et al. 1997), or even at the macro-level (Elipe \& Lantada 2012); the only requirement is the right combination of the geometry and of the deformation mechanism. Hence, a variety of auxetic materials and structures can be investigated (Evans \& Alderson 2000).

A simple distributed compliant mechanism design problem, see in particular Sigmund (1997) and Nishiwaki et al. (1998), is formulated to handle auxetic structures made of Cosserat media. The SIMP-like model t is applied to interpolate the constitutive tensor of the micropolar solid depending on the unknown material density (Bendsøe \& Kikuchi 1988).

The layout of this paper is as follows. The equilibrium equation for linear elastic isotropic micropolar solids is recalled in its strong formulation (Section 2.1), and in its weak formulation discretized by displacementbased finite elements (Section 2.2). The topology optimization problem for the synthesis of distributed compliant mechanisms is presented in Section 3 formulating remarks on the strategies employed to compute sensitivity of the objective function in an efficient way. Some applications illustrate the performances of the optimization algorithm in Section 4 and the crucial role played by the material internal length. The main results of the paper are summarized in Section 5, along with ongoing developments and future perspectives.

\section{Problem formulation}

\subsection{Strong formulation}

Micropolar elasticity is a continuum theory that resorts to additional degrees of freedom (i.e. microrotations) with respect to classical elasticity. These degrees of freedom are especially conceived to describe some features of the deformation of materials endowed with a fibrous or cellular microstructure.

Following Bruggi \& Taliercio (2012), a body made of any micropolar material that occupies a $2 \mathrm{D}$ domain $\Omega$ referred to an orthogonal Cartesian reference system is considered in this section. Note that, in the following, latin indices will be assumed to range over 1 and 2 and summation over repeated indices will be implied.

Being the solid under study a micropolar solid, the kinematics of its material particles can be fully characterized by a displacement field, $\underline{u}$, and a microrotation field, $\underline{\omega}$, both depending on the position and on the normal $(\underline{n})$ to the surface element on which they act.

The strain state of the $2 \mathrm{D}$ solid can be, then, defined through micropolar strains $\epsilon_{i j}$ and microcurvatures $\kappa_{i 3}$, with: 
$\epsilon_{i j}=u_{j, i}-\epsilon_{i j} \omega_{3}, \kappa_{i 3}=\omega_{3, i}$,

where $\epsilon_{i j}$ is the $2 \mathrm{D}$ permutation symbol and $\omega_{3}$ is the only significant component of the microrotation field.

Because of the need to describe also the interaction between the material particles, traction vector $\underline{t}$ and couple traction $\underline{c}$ are, here, introduced. Note that in the $2 \mathrm{D}$ case, $\underline{c}$ has only one nonvanishing component, $c_{3}$.

Denoting by $\underline{\underline{\sigma}}$ and $\underline{\underline{m}}$ the force stress and couple stress tensors, respectively, such that $\underline{t}=\underline{\sigma}^{T} \underline{n}, \underline{c}=$ $\underline{\underline{m}}^{T} \underline{n}$, the equilibrium equations for an infinitesimal $2 \mathrm{D}$ material element not subjected to body forces or body couples can be written as:

$\sigma_{i j, i}=0, \quad m_{i 3, i}+\epsilon_{k l} \sigma_{k l}=0 \quad$ in $\Omega$.

In order to complete the equilibrium equations, boundary conditions must be defined. The boundary of the domain, $\Gamma$, is divided into four parts, $\Gamma=\Gamma_{t} \cup \Gamma_{c} \cup$ $\Gamma_{u} \cup \Gamma_{\omega}$ depending on the different kind of boundary conditions: tractions $\underline{t}_{0}\left[\mathrm{~F} / \mathrm{L}^{2}\right]$ are applied on $\Gamma_{t}$, couples $\underline{c}_{0}\left[\mathrm{FL} / \mathrm{L}^{2}\right]$ on $\Gamma_{c}$, prescribed displacements $\underline{u}_{0}$ on $\Gamma_{u}$ and prescribed microrotations $\underline{\omega}_{0}$ on $\Gamma_{\omega}$.

The static (Neumann) boundary conditions, under vanishing couples, read:

$\sigma_{i j} n_{i}=t_{0 j}$ on $\Gamma_{t}, \quad m_{i 3} n_{i}=0$ on $\Gamma_{c}$,

while the kinetic boundary conditions (Dirichelet) are:

$\underline{u}=\underline{u}_{0}$ on $\Gamma_{u}, \quad \underline{\omega}=\underline{\omega}_{0}$ on $\Gamma_{\omega}$.

As shown in de Borst \& Sluys (1991), the constitutive equations for isotropic linearly elastic micropolar centrosymmetric materials under plane strain conditions can be expressed as:

$\sigma_{i j}=\lambda \epsilon_{r r} \delta_{i j}+\left(\mu+\mu_{c}\right) \epsilon_{i j}+\left(\mu-\mu_{c}\right) \epsilon_{j i}$,

$m_{i 3}=2 \mu \ell^{2} \kappa_{i 3}$,

where $\lambda, \mu$ and $\mu_{c}$ are generalized Lamé constants (with $\left.\mu, \mu_{c}, \lambda+\mu>0\right)$ and $\ell(>0)$ is a characteristic material length that governs the intrinsic material bending stiffness.

Closed-form estimates of the characteristic length of a micropolar material are provided e.g. in de Borst \& Sluys (1991), depending on the shape and the size of its grain. Experimental investigations on the micropolar behavior of a wide range of materials, reported in Lakes (1995), show that the characteristic length of many composites is of the same order of the microstructural size. However, in materials as graphite and human bone, the parameter $\ell$ can exceed the cell dimension. In Lakes (1993) the possibility to create strongly
Cosserat elastic materials with characteristic length $\ell$ much greater than the structure size is taken into account e.g. for open cell foams.

The characteristic length $\ell$ has not to be necessarily seen as a parameter related to the material microstructure. As introduced in Section 1, curved beam-like material distributions replace truss-like solutions typical of Cauchy solids in minimum compliance design, depending on the intrinsic material bending stiffness provided by $\ell$. A suitable tuning of this parameter can be therefore operated with the aim of switching between these two sets of optimal solutions.

Usually, alternative 'engineering' micropolar constants, that can be determined though simple tests, are introduced (see e.g. Gauthier \& Jahsman 1975; Lakes 1995). The expressions of these constants in terms of generalized Lamé constants read:

$-E=\mu(3 \lambda+2 \mu) /(\lambda+\mu)=$ Young's modulus,

$-\nu=\lambda /(2 \lambda+2 \mu)=$ Poisson's ratio,

$-N=\sqrt{\mu_{c} /\left(\mu+\mu_{c}\right)}=$ coupling number.

Note that $E, \nu$ and $N$ must fulfil some constraints in order to guarantee the positive-definiteness of the strain energy density. In the $2 \mathrm{D}$ case these constraints read:

$E>0, \quad|\nu|<1, \quad 0 \leq N \leq 1$.

$N$ is a measure of the coupling between microrotations and macrorotations. For $N=0$ no coupling arises and the solution of the micropolar elastic equilibrium matches classical elasticity, if $\ell=0$. For $N=1$ these fields are perfectly coupled, meaning that independent kinematical degrees of freedom do not include microrotations anymore. Many of the effects occuring in a micropolar solid are maximized in this case, see in particular the stiffening of thin elements in bending and the reduction of stress peaks around holes (Lakes 1985). Strongly Cosserat elastic materials exhibiting large coupling numbers are discussed e.g. in Lakes (1993).

By replacing Eqn. (1) in Eqn. (5) and, then, in Eqn. (2), the strong formulation of the static equilibrium equations in terms of displacements and microrotations are derived:

$$
\begin{aligned}
& \left(\lambda+\mu-\mu_{c}\right) u_{i, i j}+\left(\mu+\mu_{c}\right) u_{j, i i}-2 \mu_{c} \in_{i j} \omega_{3, i}=0 \\
& 2 \mu \ell^{2} \omega_{3, i i}+\mu_{c} \in_{i j}\left(u_{j, i}-u_{i, j}\right)-4 \mu_{c} \omega_{3}=0 .
\end{aligned}
$$

In order to complete the strong formulation of the problem, the static boundary conditions (3) are, then, rewrit- 
ten in terms of kinematic variables as:

$\lambda u_{r, r} n_{j}+\left[\left(\mu+\mu_{c}\right) u_{j},{ }_{i}+\left(\mu-\mu_{c}\right) u_{i},{ }_{j}\right] n_{i}=t_{0 j}$,

$2 \mu \ell^{2} \omega_{3, i} n_{i}=0$.

\subsection{Weak formulation and problem discretization}

The weak formulation for the problem defined by Eqs. $(4,7,8)$ is, here, derived in order to apply the displacementbased finite element method.

As usually done, the strong form of the equilibrium equations (7) is tested with suitable displacements, $\underline{v}$, and rotation, $\psi_{3}$, weighting functions and integrated in the domain.

The problem can be, then, reformulated as: find $\underline{\widetilde{u}}(t)=\left\{\underline{u}(t), \omega_{3}(t)\right\}$ with $\underline{u}(t), \omega_{3}(t) \in H^{1}$ such that $\left.\underline{u}\right|_{\Gamma_{u}}=\underline{u}_{0},\left.\omega_{3}\right|_{\Gamma_{\omega}}=\omega_{30}$ and

$$
\begin{aligned}
& \int_{\Omega}\left[A_{i j k l} \epsilon_{i j}(\underline{\widetilde{u}}) \epsilon_{k l}(\underline{\widetilde{v}})+2 \mu_{c}\left(\epsilon_{12}(\underline{\widetilde{u}})-\epsilon_{21}(\underline{\widetilde{u}})\right) \psi_{3}\right] d \Omega \\
& =\int_{\Gamma_{t}} \underline{t}_{0} \cdot \underline{v} d \Gamma, \\
& \int_{\Omega}\left[2 \mu \ell^{2} \kappa_{i 3}\left(\omega_{3}\right) \kappa_{i 3}\left(\psi_{3}\right)-2 \mu_{c}\left(\epsilon_{12}(\underline{\widetilde{u}})-\epsilon_{21}(\underline{\widetilde{u}})\right) \psi_{3}\right] d \Omega \\
& =0
\end{aligned}
$$

$\forall \underline{v}(t)=\left\{\underline{v}(t), \psi_{3}(t)\right\}$ with $\underline{v}(t), \psi_{3}(t) \in H^{1}, \forall t \in[0, T]$, where:

$$
A_{i j k l}=\lambda \delta_{i j} \delta_{h k}+\left(\mu+\mu_{c}\right) \delta_{i h} \delta_{j k}+\left(\mu-\mu_{c}\right) \delta_{i k} \delta_{j h} .
$$

The problem (9) here derived is, then, discretized in order to approximate, through FEM, the displacement and rotation fields. Quadrangular elements with bi-linear shape functions (see e.g. Providas \& Kattis 2002; Sharbati \& Naghdabadi 2006) are employed.

\section{The topology optimization problem}

\subsection{Problem formulation}

Classical formulations of topology optimization distribute a prescribed amount of isotropic material in order to minimize the so-called structural compliance, which is the work of the external forces at equilibrium (Bendsøe \& Sigmund 2003). Minimizing the structural compliance is, in turn, equivalent to minimize the overall elastic strain energy, that means looking for the stiffest way to bear the load.

Alternatively, formulations of topology optimization can be adopted to perform the synthesis of compliant mechanisms, i.e. flexible mechanisms that transfer an input force or displacement to another point through elastic body deformation. Optimal design can be addressed finding the distribution of material that maximizes the output displacement $u_{\text {out }}$ applied to some portion of a device connected to the structure for an input displacement enforced elsewhere through an actuator (Sigmund 1997; Nishiwaki et al. 1998).

Assuming, for simplicity's sake, that the input actuator is a linear strain based actuator, it can be modeled resorting to a spring with stiffness $k_{i n}$. The output workpiece is modeled through a spring with stiffness $k_{\text {out }}$, herein equal to $k_{i n}$. A lumped compliant mechanism exploits rotations at the joints of members that are almost rigid. Since rigid members can only store a limited amount of energy, the flexural regions are increasingly subject to concentrated stress. Alternatively, a distributed compliant mechanism exploits most of its material to store elastic energy; thus, the stress is more evenly distributed throughout the overall mechanism. Assuming low stiffness of the input/output springs with respect to the domain to be optimized, quasi-lumped compliant mechanisms arise, see e.g. Figure 2(a). Increasing such a parameter, stiff structures are found that maximize the output displacement $u_{\text {out }}$ as distributed compliant mechanisms with no articulation, see e.g. Figure 2(b) and Figure 6(a). The latter setup is employed in the following setting to maximize $u_{\text {out }}$ to deal with the optimal design of auxetic structures, see e.g. Figure 6(b).

The discrete version of the topology optimization problem can be obtained dividing the design domain into $n$ finite elements and assigning a design variable $x_{e}$ to the $e$-th element. Accordingly, the problem can be stated as:

$$
\left\{\begin{array}{cl}
\max _{x_{m i n}<x_{e}<1} u_{\text {out }} \\
\text { s.t. } & {\left[\begin{array}{cc}
\mathbf{K}_{u u}+\mathbf{K}_{s} & \mathbf{K}_{u \omega} \\
\mathbf{K}_{u \omega}^{T} & \mathbf{K}_{\omega \omega}
\end{array}\right]\left\{\begin{array}{c}
\mathbf{U}_{u} \\
\mathbf{U}_{\omega}
\end{array}\right\}=\left\{\begin{array}{c}
\mathbf{F} \\
\mathbf{0}
\end{array}\right\},} \\
& \frac{1}{V} \sum_{N} x_{e} V_{e} \leq V_{f} .
\end{array}\right.
$$

In the above statement, Eqn.(11) 2 enforces the discrete equilibrium of the micropolar solid under the assumptions of linear modeling. The adoption of a linear model can be a valid simplification in case of distributed compliant mechanism and structures that undergo small displacements and deformations, as considered in the application of the herein investigated auxetic structures. In the case of quasi-lumped compliant mechanisms, results achieved through the linear model provide only some preliminary advise towards the achievement of an optimal solution. Indeed, the synthesis of effective mechanisms that undergo large displace- 
ments should account for geometrical non-linearity, see in particular Pedersen et al. (2001).

$\mathbf{U}_{u}$ and $\mathbf{U}_{\omega}$ denote the sub-vector of displacements and micro-rotations, respectively, within the vector of nodal unknowns $\mathbf{U}$. Blocks $\mathbf{K}_{u u}, \mathbf{K}_{u \omega}$ and $\mathbf{K}_{\omega \omega}$ of the stiffness matrix and block $\mathbf{F}$ of the vector of the design independent external forces can be easily recovered from Eqn. (9). $\mathbf{K}_{s}$ is a diagonal matrix made of zeros except for the entries referring to the input and the output displacements, where it takes the values $k_{i n}$ and $k_{\text {out }}$, respectively.

The blocks of the stiffness matrix depend on the variables of the element-wise constant density discretization according to the SIMP model (Bendsøe \& Kikuchi 1988). One has $E\left(x_{e}\right)=x_{e}^{p} \bar{E}$, hereafter with $p=3$. A lower bound $x_{\min }>0$ is enforced on each $x_{e}$ to prevent singularities in the stiffness matrix.

Finally, Eqn. (11) $)_{3}$ enforces the volume constraint, being $V$ the volume of the design domain, $V_{e}$ the volume of the $e$-th element and $V_{f}$ the prescribed admissible volume fraction.

The conventional density filter (Bourdin 2001; Bruns \& Tortorelli 2001) is implemented in the numerical simulations to sketch optimal auxetic structures using a simple approach that is conceived for distributed compliant mechanisms. The original design variables $x_{e}$ are replaced by a new set of physical unknowns $\tilde{x}_{e}$ that are computed as a weighted sum of the densities of the neighborhood elements lying within the circular domain of radius $r_{\min }$.

Dealing with quasi-lumped compliant mechanisms, one should additionally prevent the arising of the socalled 'finite element hinges' where most of the deformation takes place among small sets of finite elements connected by one node (Pedersen et al. 2001). As deeply investigated in Sigmund (2007) and Wang et al. (2011), approaches as the herein adopted density filter and the Heaviside projection method (see e.g. Guest et al. 2004) are not sufficient to cope successfully with this issue: they provide mesh-independence at a global level (the overall topology is preserved with mesh refinement), but fail in ensuring a local mesh-independence of small features as 'hinges'.

To ensure a full mesh-independence, the modified robust topology optimization formulation proposed by Wang et al. (2011) should be implemented, re-writing Eqn. (11) as a min-max problem that combines an erosion, intermediate and dilation projection: at each iteration, the micropolar equilibrium of Eqn. (11) 2 should be solved for each one of the three designs.

\subsection{Sensitivity computation}

The problem in Eqn. (11) is solved via mathematical programming, adopting the Method of Moving Asymptotes (MMA) (Svanberg 1987) as minimizer. To this purpose, at each iteration the sensitivity computation of the objective function is required. To speed up the computation, the adjoint method is adopted, see e.g. Liu \& Tovar (2014). The output displacement $u_{\text {out }}$ can be re-written as

$u_{\text {out }}=\left\{\begin{array}{ll}\mathbf{L}^{T} & \mathbf{0}\end{array}\right\}\left\{\begin{array}{c}\mathbf{U}_{u} \\ \mathbf{U}_{\omega}\end{array}\right\}$,

where $\mathbf{L}$ is a vector made of zeros except for the entry referring to the output displacement $u_{\text {out }}$ where it takes unitary value. The objective function does not change if one adds at the right hand side of the above equation a zero function that involves the discrete linear equilibrium for micropolar solids reported in Eqn. $(11)_{2}$ :

$-\left\{\begin{array}{ll}\boldsymbol{\lambda}_{u}^{T} & \boldsymbol{\lambda}_{\omega}^{T}\end{array}\right\}\left(\left[\begin{array}{cc}\mathbf{K}_{u u}+\mathbf{K}_{s} & \mathbf{K}_{u \omega} \\ \mathbf{K}_{u \omega}^{T} & \mathbf{K}_{\omega \omega}\end{array}\right]\left\{\begin{array}{c}\mathbf{U}_{u} \\ \mathbf{U}_{\omega}\end{array}\right\}-\left\{\begin{array}{c}\mathbf{F} \\ \mathbf{0}\end{array}\right\}\right)$,

where $\left\{\boldsymbol{\lambda}_{u}^{T} \boldsymbol{\lambda}_{\omega}^{T}\right\}$ is any arbitrary but fixed vector. After rearrangement of terms, the derivative of the objective function with respect to the $j$-th unknown may be computed as:

$\frac{\partial u_{o u t}}{\partial x_{j}}=-\left\{\boldsymbol{\lambda}_{u}^{T} \boldsymbol{\lambda}_{\omega}^{T}\right\}\left[\begin{array}{cc}\frac{\partial \mathbf{K}_{u u}}{\partial x_{j}} & \frac{\partial \mathbf{K}_{u \omega}}{\partial x_{j}} \\ \frac{\partial \mathbf{K}_{u \omega}^{T}}{\partial x_{j}} & \frac{\partial \mathbf{K}_{\omega \omega}}{\partial x_{j}}\end{array}\right]\left\{\begin{array}{c}\mathbf{U}_{u} \\ \mathbf{U}_{\omega}\end{array}\right\}$,

where $\left\{\boldsymbol{\lambda}_{u}^{T} \boldsymbol{\lambda}_{\omega}^{T}\right\}$ satisfies the adjoint equation:

$\left[\begin{array}{cc}\mathbf{K}_{u u}+\mathbf{K}_{s} & \mathbf{K}_{u \omega} \\ \mathbf{K}_{u \omega}^{T} & \mathbf{K}_{\omega \omega}\end{array}\right]\left\{\begin{array}{c}\boldsymbol{\lambda}_{u} \\ \boldsymbol{\lambda}_{\omega}\end{array}\right\}=\frac{\partial u_{o u t}}{\partial \mathbf{U}}=\left\{\begin{array}{l}\mathbf{L} \\ \mathbf{0}\end{array}\right\}$.

Denoting by $\mathbf{k}_{-, e}$ a generic block of the stiffness matrix of the $e^{- \text {th }}$ element, one has that $\partial \mathbf{k}_{-, e} / \partial x_{j}=$ $\delta_{e j} p x_{e}^{(p-1)} \overline{\mathbf{k}}_{-, e}$, where $\delta_{e j}$ is the Kronecker delta and $\overline{\mathbf{k}}_{-, e}$ is the relevant stiffness computed for full material density.

It must be also remarked that the use of a density filter implies a chain rule modification of the sensitivities of the objective function $u_{\text {out }}$ and the volume constraint, to compute the derivatives with respect to the physical unknowns $\tilde{x}_{j}$ instead of the original one $x_{j}$. 


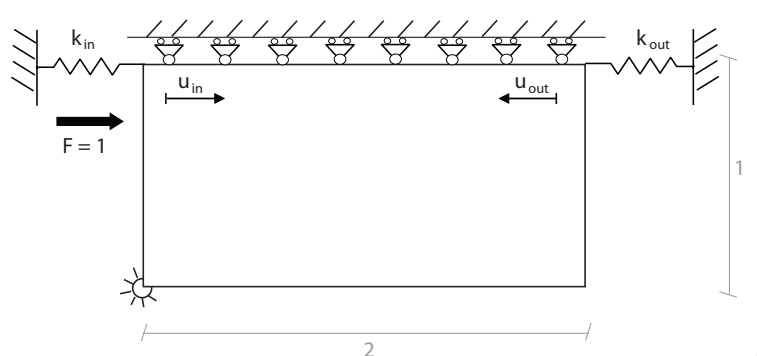

(a)

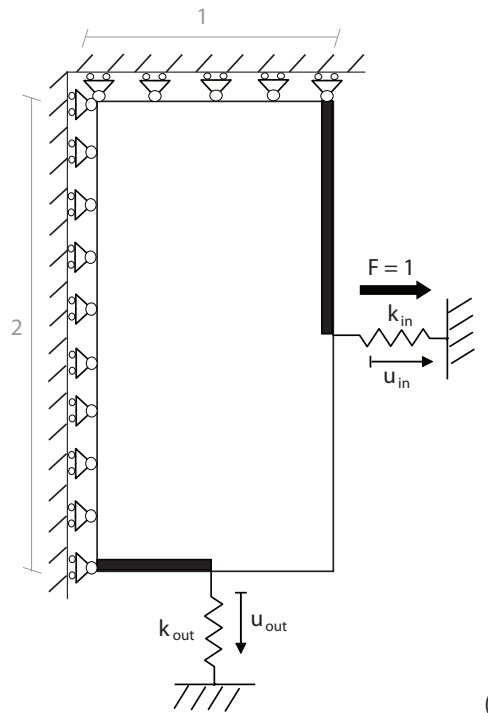

(b)

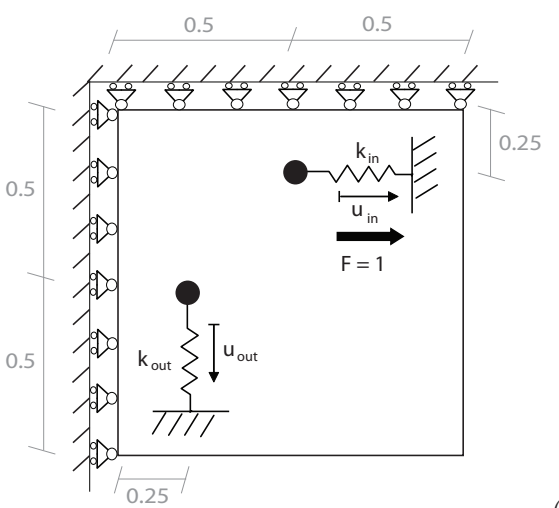

(c)

Fig. 1 Geometry and boundary conditions: Example 1 (a), Example 2 (b) and Example 3 (c).

\section{Numerical results}

In this section a few optimal layouts of distributed compliant mechanisms made of Cosserat materials are investigated and the performances of the proposed numerical scheme are assessed. Moreover, a comparison with conventional solutions based on the Cauchy model is shown.

A set of numerical simulations with different values of $\ell$ are carried out for each example to investigate the effect of the characteristic length of the material on the optimal material layouts of micropolar solids.

As introduced in Section 1 and detailed in Section $2.1, \ell$ is strictly related to the size of the microstructure. According to Eqn. $(5)_{2}$, this material parameter affects the magnitude of couples stresses that arise from the microcurvatures field, providing a measure of the additional flexural stiffness observed through the micropolar model. Thin plates and beams made of micropolar material are stiffer than those described through conventional kinematics, whereas no difference is observed for thick structures. This size effect allows measuring the characteristic length of a microstructured material from experiments performed on specimens of different size.

Alternatively, $\ell$ can be used as a tuning parameter to enhance flexural stiffness of Eqn. $(5)_{2}$ with respect to the contribution in Eqn. (5) 1 . Big values of $\ell$ can be artificially adopted to steer the minimizer towards curve-like optimal layouts.

In all the following examples, a micropolar material with the following reference properties will be employed if not differently specified:

$\bar{E}=1000 \mathrm{~N} / \mathrm{m}^{2} \quad \nu=0.2 \quad N=0.5$.

Numerical investigations are performed according to Eqn. (11): the admissible volume fraction $V_{f}$ is considered equal to 0.25 in the first example and equal to 0.1 in the last two examples, while $r_{\min }=2.0 d_{m}$ for all of them. Note that $d_{m}$ is the reference size of the square elements of the adopted meshes.

All the optimal layouts are shown in terms of the physical unknowns $\tilde{x}_{e}$. Table 1 reports the absolute value of the input displacement $u_{i n}$, the absolute value of the output displacement $u_{\text {out }}$ and their ratio $u_{\text {out }} / u_{\text {in }}$. A comparison of the results is made by focusing on the non-dimensional parameter $\ell / b$ defined as the ratio of the characteristic material length $\ell$ to the reference dimension of the domain $b$. This ratio can be considered as a suitable measure of the influence of the Cosserat effects on the computed optimal topologies. Indeed, experimental results on micropolar plates and beams in bending are generally investigated in terms of maximum displacement vs. non-dimensional parameter $\ell / b$ to pinpoint the characteristic size effect. For $\ell / b \rightarrow 0$ the size of the microstructure is too small to affect the overall structural behavior, whereas larger values of this ratio are generally related to non-negligible micropolar effects. Strong Cosserat effects are found when the dimension of the microstructure approaches that of the structural element, or $\ell / b>1$ is artificially used to simulate a material with prevailing flexural stiffness. 
Numerical simulations are performed assuming a reference value $b=1 \mathrm{~m}$. Due to the liner elastic modeling, the achieved results can be easily scaled to other problem sizes.

\subsection{Example 1: the inverter}

The formulation presented in Section 3 is preliminary assessed addressing the benchmark example in Figure 1(a). Only half of the domain is considered to design an optimal device that maximizes the leftward displacement $u_{\text {out }}$ for an applied rightward force $F=1 N$. Figure 2 shows the optimal design achieved adopting a Cauchy material model $(N=0$ and $\ell=0)$ and enforcing two different values for the stiffness of the input and output springs. Figure 2(a) refers to the case $k_{\text {in }}=k_{\text {out }}=k=0.01 \mathrm{~N} / \mathrm{m}$, whereas Figure 2(b) refers to $k_{\text {in }}=k_{\text {out }}=k=0.1 \mathrm{~N} / \mathrm{m}$. Both layouts are in full agreement with the benchmark solution originally reported in Sigmund (1997). For the smaller value of $k$, a quasi-lumped mechanism is found where main parts are connected by thin regions playing as 'hinges'. However, it must be remarked that the minimum thickness of these 'hinges' can't be effectively controlled by the adopted density filter, see Section 3.1. For the larger value of $k$ a distributed mechanism is found, meaning that no 'hinge' appear and the strain is more evenly spread throughout the overall optimal design. Further examples of distributed compliant mechanisms may be seen e.g. in Figure 6, showing optimal layouts whose members show approximately the same thickness and no articulation is found.

Since the main goal of this contribution is the achievement of auxetic structures through the optimal design of distributed compliant mechanisms, the setup $k_{\text {in }}=$ $k_{\text {out }}=k=0.1 \mathrm{~N} / \mathrm{m}$ will be preserved in all the subsequent simulations.

Figure 3 shows the optimal layouts achieved enforcing the micropolar model for different values of the characteristic length of the material. For $\ell$ that is one hundredth of the size of the design domain, see Figure $3(\mathrm{a})$, no noticeable difference arises with respect to the Cauchy-based solution represented in Figure 2(b). Increasing the value of the characteristic length to $\ell / b=$ 0.1 , the main truss-like component of the optimal mechanism takes a more rounded layout, see Figure 3(c), whereas for $\ell / b=0.05$ an intermediate layout is found. Adopting materials with $\ell>0$ means providing additional flexural stiffness with respect to the conventional Cauchy-based model. A reduced deformability is observed, see Table 1 . This is in agreement with results reported for the minimum compliance formulation, see e.g. Veber \& Taliercio (2011). To perform the maximization of the output displacement, regions connecting the 45-degree inclined bars to the remaining part of the design get thinner for increasing values of $\ell$. The arising of 'hinges' is expected when enforcing $\ell / b>0.1$ to transform the Cauchy-based distributed mechanism in a quasi-lumped one. The approach proposed by Wang et al. (2011) should be implemented to robustly handle this kind of layouts, see Section 3.1.

An additional simulation is performed to investigate optimal layouts in case of strongly Cosserat elastic materials with large coupling number $N$. Figure 4 shows the optimal layout found for $N=0.9$ and $\ell / b=0.05$. The achieved optimal layout resembles that of Figure 3 (c) for $N=0.5$ and $\ell / b=0.1$. As already reported in Bruggi \& Taliercio (2012), it may be concluded that optimal layouts achieved for large coupling numbers emphasize micropolar effects that are mainly governed by the characteristic length of the material $\ell$.

Table 2 reports the output displacement $u_{\text {out }}$ computed for the above optimal layouts assuming different sets of parameters for the micropolar material. A crosscheck confirms that each design performs better than the others for the couple $(\ell / b, N)$ it has been designed for: the relevant $u_{\text {out }}$ is the maximum among the values of each column. Hence, it is worth accounting for the microstructure of the material to get an ad hoc design that maximizes the target performance depending on the material length.

Reading the table by row, different trends can be reported referring to the effect of a variation of the material characteristic length for the same design. Looking at the layouts achieved for $\ell / b=0$ and $\ell / b=0.01, u_{\text {out }}$ decreases when increasing $\ell / b$; looking at the layouts achieved for $\ell / b=0.05$ and $\ell / b=0.1, u_{\text {out }}$ is maximum for an intermediate value of the ratio $\ell / b$. In both cases, the adoption of a larger $N$ provides bigger output displacements. Of course, these trends are strictly dependent on the features of the considered topology and can not be easily generalized.

It must be remarked that values reported in Table 1 and 2 can be used only for a preliminary comparison of the sketched layouts, which should be regarded by the designer as inspiring solutions. Performances of the optimal layouts should be investigated referring to their engineered versions accounting, among the others, for manufacturing constraints.

Finally, Figure 5 compares the history plot of the objective function for the optimal layouts in Figures 2(b), 3(b) and 4. The three problems converge. Being the optimization procedure initialized enforcing full density of the material all over the domain, in the very first iterations, a positive (rightward) output displacement is found instead of the expected negative (leftwards) one. 

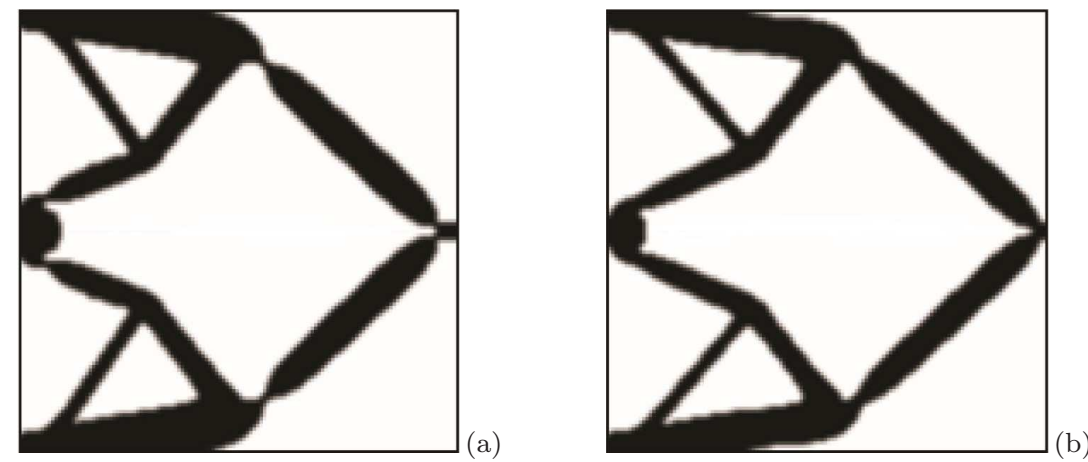

Fig. 2 Example 1: optimal design with Cauchy material for $k_{\text {in }}=k_{\text {out }}=k=0.01 \mathrm{~N} / \mathrm{m}$ (a) and $k_{\text {in }}=k_{\text {out }}=k=0.1 \mathrm{~N} / \mathrm{m}(\mathrm{b}-$ $u_{\text {out }}=0.84 \mathrm{~mm}$ )
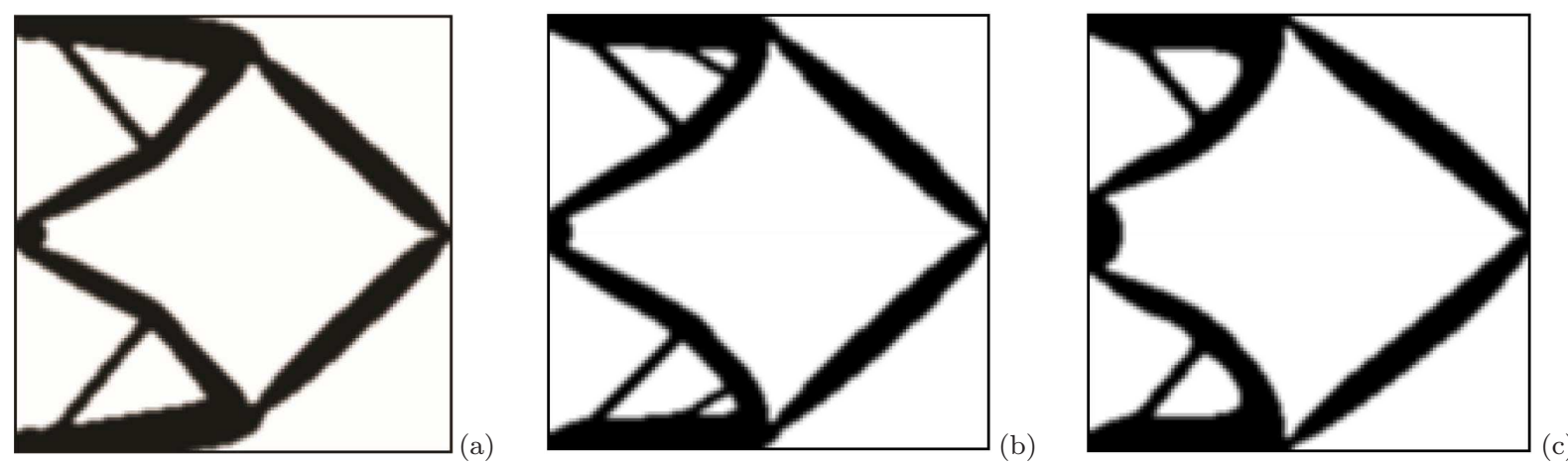

Fig. 3 Example 1: optimal design with micropolar material $N=0.5$ and $\ell / b=0.01\left(\mathrm{a}-u_{\text {out }}=0.83 \mathrm{~mm}\right), \ell / b=0.05\left(\mathrm{~b}-u_{\text {out }}=0.81\right.$ $\mathrm{mm})$ and $\ell / b=0.1\left(\mathrm{c}-u_{\text {out }}=0.65 \mathrm{~mm}\right)$.

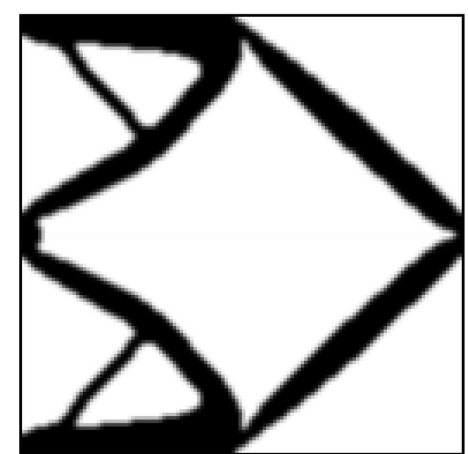

Fig. 4 Example 1: optimal design with micropolar material for $N=0.9$ and $\ell / b=0.05\left(u_{\text {out }}=0.87 \mathrm{~mm}\right)$.

Adopting as a reference the Cauchy-based optimization, increased micropolar effects are introduced through the enforcement of $\ell>0$ and to the adoption of a larger $N$ for the same value of the characteristic length of the material. For $\ell>0$ the number of iterations to converge increases, especially for $N=0.9$.

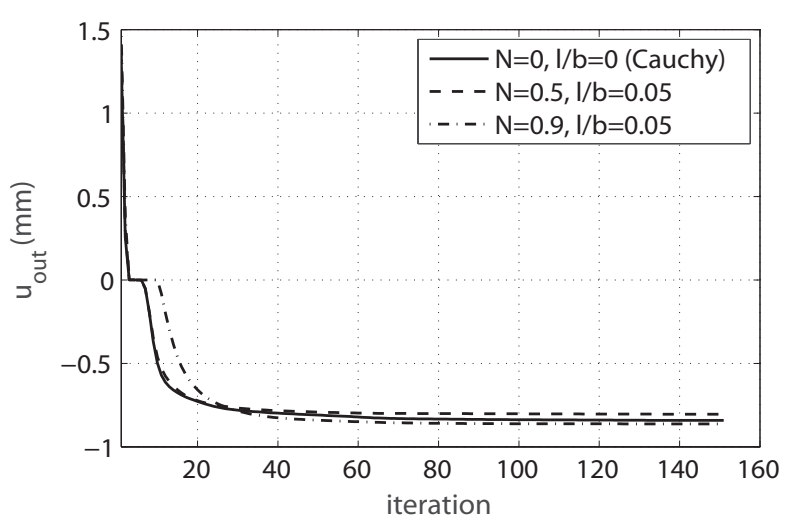

Fig. 5 Example 1: history plot of the objective function for the optimal layouts in Figures 2(b), 3(b) and 4.

\subsection{Example 2: auxetic structures in a rectangular} domain

This second example focuses on the synthesis of auxetic structures through the adoption of the formulation for the design of distributed compliant mechanisms that is stated in Eqn.(11). 

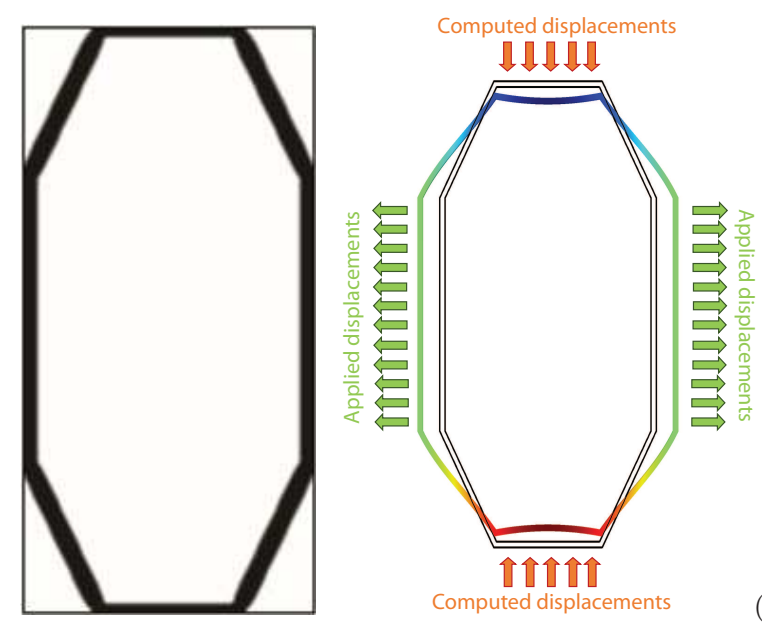

(a)
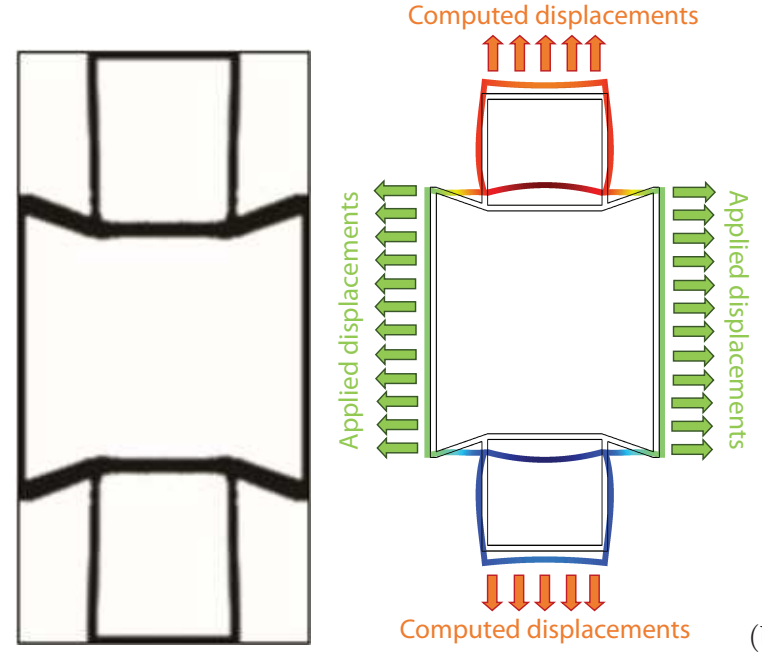

Fig. 6 Example 2: optimal design with Cauchy material: non-auxetic $\left(\mathrm{a}-u_{\text {out }}=1.21 \mathrm{~mm}\right)$ and auxetic structure $\left(\mathrm{b}-u_{\text {out }}=1.16\right.$ $\mathrm{mm})$.

Geometry and boundary conditions represented in Figure 1(b) are considered, as inspired by an application in the field of MEMS: the design of an auxetic micro-actuator. Black regions stand for fixed zones of full material.

In the following simulations, only a quarter of the domain is handled to design optimal structures that maximize the upward or downward displacement $u_{\text {out }}$ for an applied rightward force $F=1 N$.

The Cauchy material model is considered first. Figure 6(a) shows the optimal layout achieved in case of maximization of the upward displacement. No 'hinge' is found in the achieved distributed compliant mechanism. A non-auxetic structure arises, meaning that exerting a traction along the horizontal direction a contraction is found along the vertical one. This structure behaves like any body made of a (conventional) material for which a positive Poisson's ratio is expected. Maximizing the downward displacement, the auxetic structure represented in Figure 6(b) is achieved. No 'hinge' tied to any strain peak arises. Exerting a traction along the horizontal direction, an expansion is found along the vertical one. This optimal structural layout behaves like a non-conventional material with negative Poisson's ratio. In fact, the achieved optimal design has geometrical features that are peculiar to engineered materials exhibiting extreme negative Poisson's ratio, see e.g. Sigmund (1994) and Xia \& Breitkopf (2015). It must be remarked that the proposed procedure applies to the optimal design of structures/structural components, whereas homogenization theory and periodicity conditions are alternatively dealt with when addressing material design.
Figure 7 reports the history plot of the objective function for the optimal layouts shown in Figure 6. Convergence is smooth in both cases, but the auxetic structure calls for an increased number of iterations to achieve the final value of the objective function.

When micropolar material is used to build the considered structures/structural components and the relevant characteristic length is non-negligible with respect to the size of the available geometrical domain, the Cosserat material model can be conveniently exploited in Eqn.(11). This is the case of MEMS devices made of poly-silicon fibers, but other applications can be foreseen involving materials that exhibit strong Cosserat effects, see Lakes (1993) and Lakes (1995).

Figure 8 shows the optimal layouts found in the case of micropolar material for $0.01 \leq \ell / b \leq 10$. The achieved layouts share the main topology already seen in Figure 6(b) for the Cauchy material model. Increasing $\ell$, rounded elements replace struts and ties in the optimal solution. Even a small characteristic length can be responsible for remarkable modifications in the optimal topology and its mechanical performance. Looking at Figure $8(\mathrm{a})$, inclined bars are found for $\ell / b=0.01$, instead of the vertical elements depicted in Figure 6(b). In this case, the output and input displacements are comparable with those of the Cauchy-based design. For higher values of the governing ratio $\ell / b$, stiffer solutions are found, see Table 1.

\subsection{Example 3: auxetic structures in a square domain}

Optimal auxetic structures are looked for in the square domain of Figure 1(c). Input and output points are defined within the bulk of the domain providing a wide 

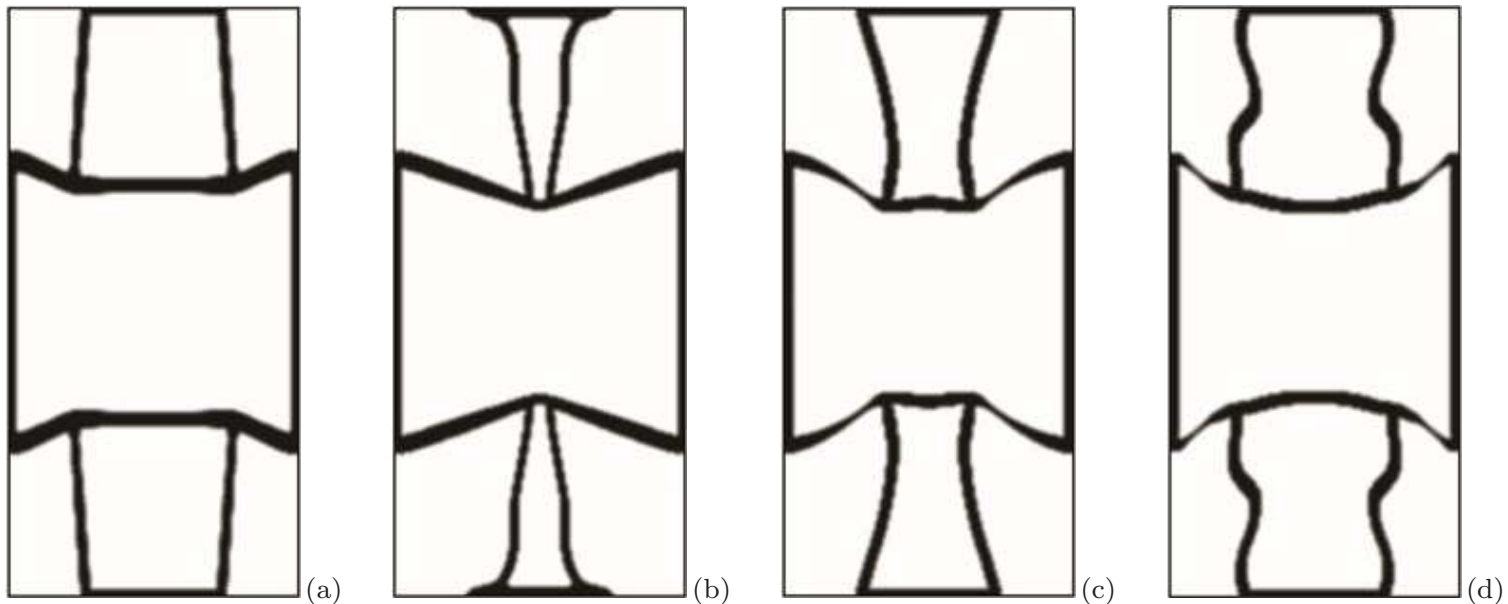

Fig. 8 Example 2: optimal design with micropolar material for $\ell / b=0.01\left(\mathrm{a}-u_{\text {out }}=1.23 \mathrm{~mm}\right), \ell / b=0.1\left(\mathrm{~b}-u_{\text {out }}=0.80 \mathrm{~mm}\right)$, $\ell / b=1\left(\mathrm{c}-u_{\text {out }}=0.34 \mathrm{~mm}\right)$ and $\ell / b=10\left(\mathrm{~d}-u_{\text {out }}=0.30 \mathrm{~mm}\right)$
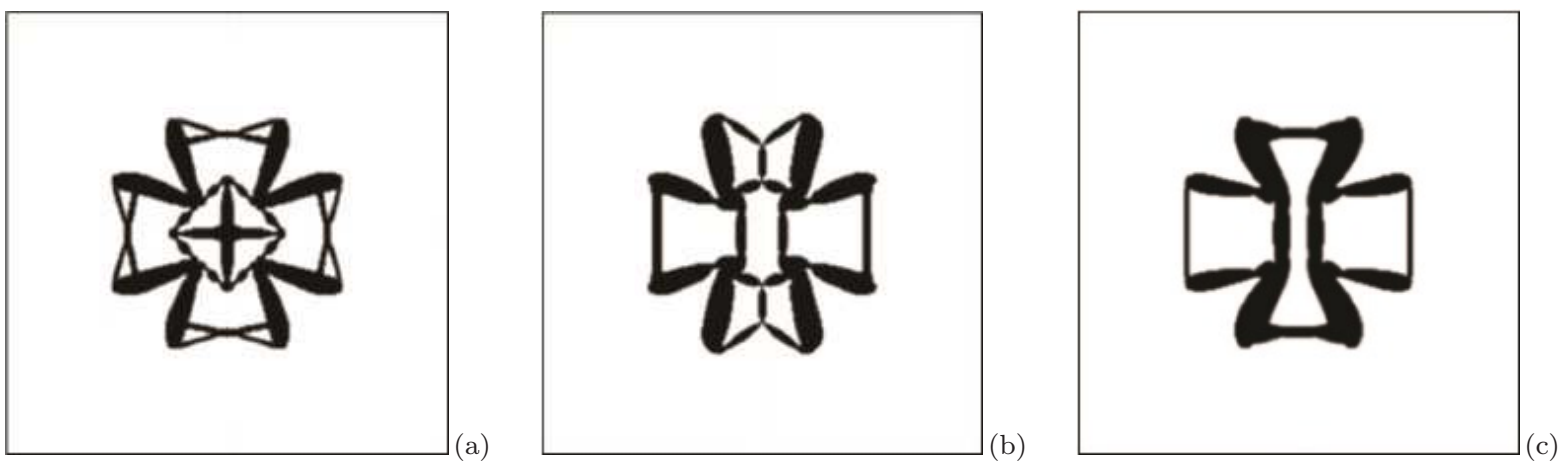

Fig. 9 Example 3: optimal design with Cauchy material $\left(\mathrm{a}-u_{\text {out }}=2.22 \mathrm{~mm}\right)$ and with micropolar material for $\ell / b=0.01(\mathrm{~b}-$ $\left.u_{\text {out }}=1.90 \mathrm{~mm}\right), \ell / b=0.015\left(\mathrm{c}-u_{\text {out }}=2.21 \mathrm{~mm}\right)$.
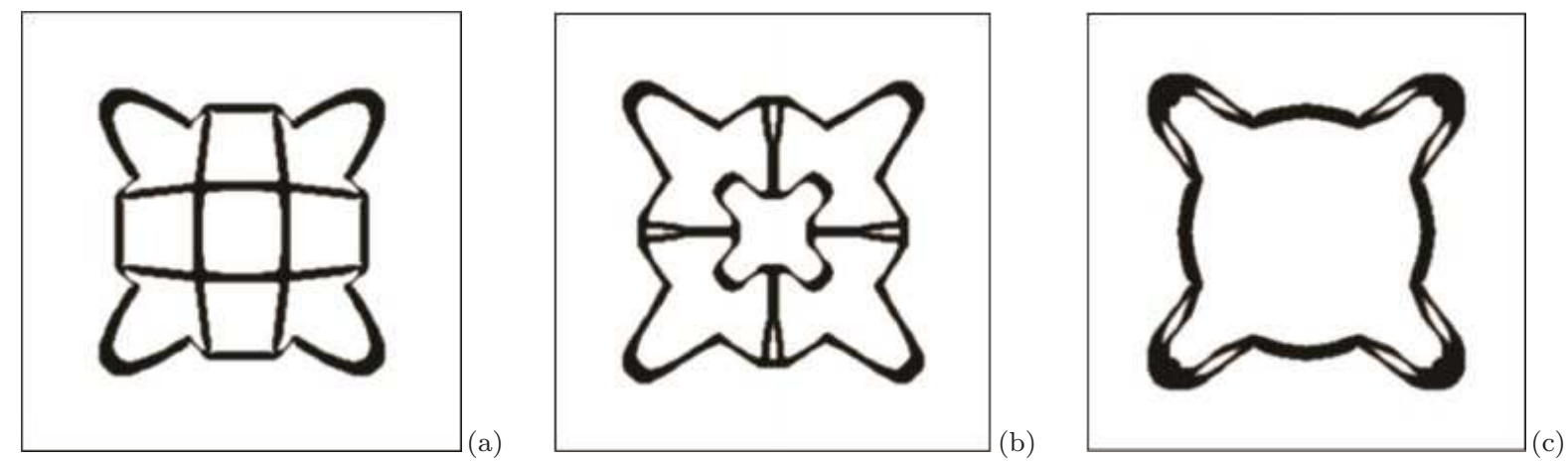

Fig. 10 Example 3: optimal design with micropolar material for $\ell / b=1\left(\mathrm{a}-u_{\text {out }}=0.48 \mathrm{~mm}\right)$, for $\ell / b=5\left(\mathrm{~b}-u_{\text {out }}=0.48 \mathrm{~mm}\right)$ and $\ell / b=10\left(\mathrm{c}-u_{\text {out }}=0.45 \mathrm{~mm}\right)$.

design region. As before, only a quarter of the domain is considered for symmetry reasons. The downward displacement $u_{\text {out }}$ is maximized for an applied rightward force $F=1 N$ using Eqn.(11).

The outcome of the simulation performed adopting the Cauchy material model is represented in Figure 9 (a). An optimal design with four symmetry axes arises. The output displacement of the auxetic structure is nearly one third of the input one.

Figure 9(b) and (c) refer to micropolar materials exhibiting a small characteristic length. An optimal design with only two symmetry axes arises in both cases. The solution shown in Figure $9(\mathrm{c})$ for $\ell / b=0.015$ is very similar to the topology found looking at the optimal design of auxetic structures in the rectangular 


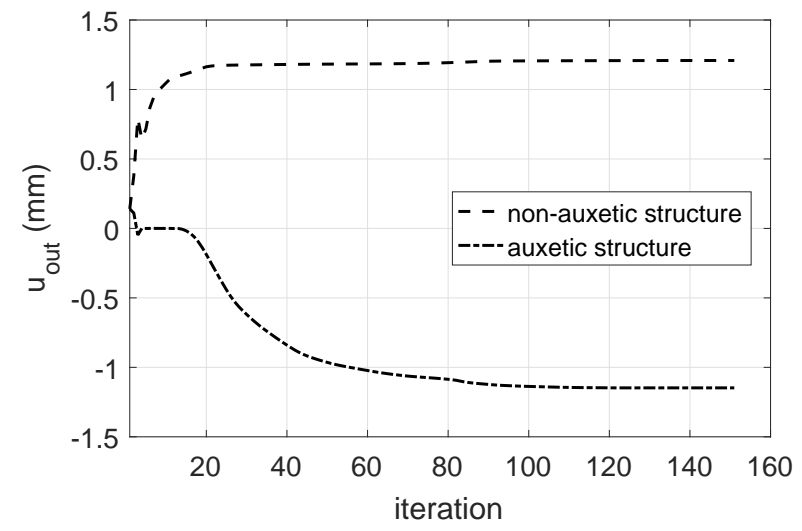

Fig. 7 Example 2: history plot of the objective function for the optimal layouts in Figure 6.

domain, see Section 4.2. As shown e.g. in Gaspar et al. (2009) addressing microstructural modeling of auxetic materials, anisotropic modifications can enhance the magnitude of the Poisson's ratio of conventional auxetic microstructures. Notwithstanding the increased flexural stiffness introduced with $\ell>0$, the output and input displacements computed for the achieved Cosseratbased layouts are comparable with those of the Cauchybased design, see Table 1.

Finally, Figure 10 shows optimal solutions computed in case of materials exhibiting strong Cosserat effects. Due to the large values of the ratio of the characteristic material length $\ell$ to the reference dimension of the domain $b$, the achieved layouts are much stiffer than the previous solutions. Symmetry with respect to four axes is preserved, as in the Cauchy-based design.

A prominent flexural stiffness is found in the pure bending-resistant layout of Figure 10(c). As originally shown by Gei et al. (2006), an analogy between the characteristic length of micropolar solids and the bending stiffness of beams can be stated. One may therefore resort to a Cosserat model with $\ell / b>1$ to generate auxetic layouts where conventional truss-like components are replaced by beam-like layouts.

\section{Conclusions}

This work investigates the adoption of topology optimization to synthesize auxetic structures and structural components made of micropolar material. A design problem is formulated to achieve distributed compliant mechanisms that undergo the deformation storing strain energy almost homogenously throughout the domain. A suitable output displacement is maximized such that, exerting a traction along a prescribed di- rection, an expansion is found along the orthogonal one. The SIMP-like model is implemented to penalize the constitutive parameters of 2D Cosserat bodies, whereas mathematical programming is applied to achieve smooth convergence of the optimization procedure. Cauchy-based layouts can be recovered by the proposed formulation, enforcing null coupling number and null characteristic length of the material.

In the literature, the auxetic behavior is generally achieved designing the material at the microstructural level. This means enforcing periodic boundary condition to a reference unit cell and exploiting homogenization to maximize the absolute value of the negative Poisson ratio of the microstructured material. As reported e.g. in Sigmund (1994) and Xia \& Breitkopf (2015), the design with negative Poisson's ratio is a challenging topic, calling for the adoption of additional constraints or relaxed forms of the objective function. Alternatively, the design approach herein proposed applies to elements made of microstructured material with prescribed properties.

This work shows that a simple formulation conceived for the design of distributed compliant mechanisms can be successfully used to sketch auxetic structures and microsystems. Since micropolar effects can be foreseen when investigating the latter kind of applications, the considered optimization problem is specialized to cope with the Cosserat material model.

Numerical simulations show that the results of the optimization are quite sensitive to the material characteristic length $\ell$. As investigated for Example 1, the same design has different output displacements depending on the material parameters used to analyze its structural response. As expected, the maximum performance is found using the material parameter it has been designed for. Hence, accounting for the microstructure of the material within the optimization problem allows to sketch ad hoc layouts that maximize the target performance depending on the material length.

More in detail, the ratio of $\ell$ to the reference dimension of the domain $b$ is a suitable measure of the influence of the Cosserat effects on the achieved optimal layouts. Even for low values of the ratio $\ell / b$, significant variations may be highlighted that are localized in a few members (Example 2) or affect the full topology (Example 3). For large values of $\ell / b$, a transition from trusslike layouts, typical of Cauchy solids, to pure bendingresistant solutions is observed, as outlined in previous investigations concerning the minimum compliance formulation. The former results could be exploited, for instance, to investigate enhanced solutions for motion conversion and resonators in MEMS applications involving poly-silicon fibers. The latter could be used 


\begin{tabular}{c|c|c|c|c|c} 
Ex. & Fig. & Material & $u_{\text {in }}$ & $u_{\text {out }}$ & $u_{\text {out }} / u_{\text {in }}$ \\
\hline 1 & $2(\mathrm{~b})$ & Cauchy & 9.18 & 0.84 & 0.09 \\
1 & $3(\mathrm{a})$ & Cosserat $(\ell / b=0.01)$ & 9.16 & 0.83 & 0.09 \\
1 & $3(\mathrm{~b})$ & Cosserat $(\ell / b=0.05)$ & 9.04 & 0.81 & 0.09 \\
1 & $3(\mathrm{c})$ & Cosserat $(\ell / b=0.1)$ & 8.95 & 0.65 & 0.07 \\
1 & 4 & Cosserat $(\ell / b=0.05)$ & 8.94 & 0.87 & 0.10 \\
\hline 2 & $6(\mathrm{a})$ & Cauchy & 9.42 & 1.21 & 0.13 \\
2 & $6(\mathrm{~b})$ & Cauchy & 6.75 & 1.16 & 0.17 \\
2 & $8(\mathrm{a})$ & Cosserat $(\ell / b=0.01)$ & 7.20 & 1.23 & 0.17 \\
2 & $8(\mathrm{~b})$ & Cosserat $(\ell / b=0.1)$ & 7.09 & 0.80 & 0.11 \\
2 & $8(\mathrm{c})$ & Cosserat $(\ell / b=1)$ & 7.67 & 0.34 & 0.04 \\
2 & $8(\mathrm{~d})$ & Cosserat $(\ell / b=10)$ & 8.09 & 0.30 & 0.04 \\
\hline 3 & $9(\mathrm{a})$ & Cauchy & 7.40 & 2.22 & 0.30 \\
3 & $9(\mathrm{~b})$ & Cosserat $(\ell / b=0.01)$ & 7.69 & 1.90 & 0.25 \\
3 & $9(\mathrm{c})$ & Cosserat $(\ell / b=0.015)$ & 8.44 & 2.21 & 0.26 \\
3 & $10(\mathrm{a})$ & Cosserat $(\ell / b=1)$ & 7.69 & 0.48 & 0.06 \\
3 & $10(\mathrm{~b})$ & Cosserat $(\ell / b=5)$ & 7.88 & 0.48 & 0.06 \\
3 & $10(\mathrm{c})$ & Cosserat $(\ell / b=10)$ & 8.00 & 0.45 & 0.06 \\
& & & &
\end{tabular}

Table 1 Examples 1-3. Comparison of the optimal layouts with $k_{i n}=k_{\text {out }}=k=0.1 \mathrm{~N} / \mathrm{m}$ in terms of input displacement $u_{i n}(\mathrm{~mm})$, output displacement $u_{\text {out }}(\mathrm{mm})$ and their ratio $u_{\text {out }} / u_{\text {in }}$.

\begin{tabular}{c|c|c|c|c|c} 
& \multicolumn{5}{|c}{$u_{\text {out }}$ computed for } \\
Optimal design & Cauchy & $\begin{array}{c}\ell / b=0.01 \\
N=0.5\end{array}$ & $\begin{array}{c}\ell / b=0.05 \\
N=0.5\end{array}$ & $\begin{array}{c}\ell / b=0.1 \\
N=0.5\end{array}$ & $\begin{array}{c}\ell / b=0.05 \\
N=0.9\end{array}$ \\
\hline Cauchy, Fig. 2(b) & 0.844 & 0.832 & 0.776 & 0.523 & 0.823 \\
$\ell / b=0.01$, Fig. 3(a) & 0.842 & 0.834 & 0.786 & 0.538 & 0.834 \\
$\ell / b=0.05$, Fig. 3(b) & 0.789 & 0.785 & 0.805 & 0.625 & 0.859 \\
$\ell / b=0.1$, Fig. 3(c) & 0.638 & 0.638 & 0.742 & 0.653 & 0.799 \\
$\ell / b=0.05$, Fig. 4 & 0.770 & 0.771 & 0.801 & 0.626 & 0.866
\end{tabular}

Table 2 Example 1. Output displacements $u_{\text {out }}(\mathrm{mm})$ computed for the optimal layouts with $k_{\text {in }}=k_{\text {out }}=k=0.1 \mathrm{~N} / \mathrm{m}$ and different assumptions on the material parameters.

to handle stiff materials exhibiting strong Cosserat effects or to generate optimal layouts whenever bendingresistant members are preferred to truss-like elements (Example 3). In both cases, the proposed formulation plays as a simple tool to inspire the designer when sketching layouts for auxetic structures.

Current research is mainly devoted to the extension of the proposed procedure to the three-dimensional framework, thus accounting for torsional Cosserat effects when designing optimal auxetic structures.

\section{References}

Anderson WB, Lakes RS (1994) Size effects due to Cosserat elasticity and surface damage in closed-cell polymethacrylimide foam. J Mater Sci 29:6413-6419

Andreassen E, Lazarov BS, Sigmund O (2014) Design of manufacturable 3D extremal elastic microstructure. Mech Mater 69:1-10
Bendsøe MP, Kikuchi N (1988) Generating optimal topologies in structural design using a homogenization method. Comput Meth Appl Mech Eng 71:197-224

Bendsøe MP, Sigmund O (1999) Material interpolations in topology optimization. Arch Appl Mech 69:635-654

Bendsøe MP, Sigmund O (2003) Topology optimization - Theory, methods and applications. Springer, New York

Bigoni D, Drugan WJ (2006) Analytical Derivation of Cosserat Moduli via Homogenization of Heterogeneous Elastic Materials. J Appl Mech 74(4):741-753

Bourdin B (2001) Filters in topology optimization. Int J Numer Methods Eng 50(9):2143-2158

Bruggi M, Taliercio A (2012) Maximization of the fundamental eigenfrequency of micropolar solids through topology optimization. Struct Multidiscip Optim 46(4):549-560

Bruns TE, Tortorelli DA (2001) Topology optimization of nonlinear elastic structures and compliant mechanisms. Comput Meth Appl Mech Eng 190(26-27):3443-3459

Cadman J, Zhou S, Chen Y, Li Q (2012) On design of multifunctional microstructural materials. J Mater Sci 48:1-16

Coelho PG, Cardoso JB, Fernandes PR, Rodrigues HC (2011) Parallel computing techniques applied to the simultaneous design of structure and material. Adv Eng Software 42:219227 
Corigliano A, De Masi B, Frangi A, Comi C, Villa A, Marchi M (2004) Mechanical characterization of polysilicon through on-chip tensile tests. J Microelectromech Syst 13(2):200-219

de Borst R, Sluys LJ (1991) Localization in a Cosserat continuum under static and dynamic loading conditions. Comput Meth Appl Mech Eng 90:805-827

Deaton JD, Grandhi RV (2014) A survey of structural and multidisciplinary continuum topology optimization: Post 2000. Struct Multidiscip Optim 49:1-38

Diaz A, Sigmund O (2010) A topology optimization method for design of negative permeability metamaterials. Struct Multidiscip Optim 41:163-177

Elipe JCA, Lantada AD (2012) Comparative study of auxetic geometries by means of computer-aided design and engineering. Smart Mater Struct 21:1-12

Eringen AC (1966) Linear theory of micropolar elasticity. J Math Mech 15:909-923

Evans KE, Alderson A (2000) Auxetic materials: Functional materials and structures from lateral thinking! Adv Mater 12(9):617-628

Fatemi J, Onck PR, Poort G, Van Keulen F (2003) Cosserat moduli of anisotropic cancellous bone: a micromechanical analysis. J Phys IV France 105:273-280

Forest S, Sab K (1998) Cosserat overall modeling of heterogeneous materials. Mech Res Commun 25(4):449-454

Forest S, Barbe F, Cailletaud G (2000) Cosserat modelling of size effects in the mechanical behaviour of polycrystals and multiphase materials. Int J Solids Struct 37(46-47):7105-7126

Gaspar N, Smith CW, Evans KE (2009) Auxetic behaviour and anisotropic heterogeneity. Acta Mater 57(3):875-880

Gauthier RD, Jahsman WE (1975) A quest for micropolar elastic constants. J Appl Mech ASME 42:369-374

Gei M, Rovati M, Veber D (2006) Effect of internal length scale on optimal topologies for Cosserat continua.IUTAM Symposium on Topological Design Optimization of Structures, Machines and Materials: Status and Perspectives, Martin P. Bendse et al.(eds) $157-166$

Guest J, Prevost J, Belytschko T (2004) Achieving minimum length scale in topology optimization using nodal design variables and projection functions. Int J Numer Methods Eng 61(2):238-254

Koskinent J, Steinwall JE, Soave R, Johnson HH (1993) Microtensile testing of free-standing polysilicon fibers of various grain sizes. J Micromech Microeng 3:13-17

Lakes RS (1985) A pathological situation in micropolar elasticity, J Applied Mechanics, 52:234-235

Lakes RS (1993) Strongly Cosserat elastic lattice and foam materials for enhanced toughness. Cellular Polymers 12(1730):157-166

Lakes RS (1995) Experimental methods for study of Cosserat elastic solids and other generalized elastic continua. In:Continuum models for materials with microstructure, Mühlhaus H. (Ed.), J. Wiley, 1-25

Larsen UD, Sigmund O, Bouwstra S (1997) Design and Fabrication of Compliant Micromechanisms and Structures with Negative Poisson's Ratio. J Microelectromech Syst 6:99-105

Levy O, Krylov S, Goldfarb I (2006) Design considerations for negative Poisson ratio structures under large deflection for MEMS applications. Smart Mater Struct 15:1459-1466

Li X, Liu Q, Zhang J (2010) A micro-macro homogenization approach for discrete particle assembly -Cosserat continuum modeling of granular materials. Int J Solids Struct 47(2):291303

Liu K, Tovar A (2014) An efficient 3D topology optimization code written in matlab. Struct Multidiscip Optim 50(6):1175-1196
Liu S, Su W (2010) Topology optimization of couple-stress material structures. Struct Multidiscip Optim 40:319?327

Mariani S, Martini R, Ghisi A, Corigliano A, Beghi M (2011) Overall elastic properties of polysilicon films: a statistical investigation of the effects of polycrystal morphology. Int $\mathrm{J}$ Multiscale Com 9(3):327-346

Nishiwaki S, Frecker MI, Min S, Kikuchi N (1998) Topology optimization of compliant mechanisms using thehomogenization method. Int J Numer Meth Engng 42:535-559

Pedersen CBW, Buhl T, Sigmund O (2001) Topology synthesis of large-displacement compliant mechanisms. Int J Numer Meth Engng 50: 2683-2705

Providas E, Kattis MA (2002) Finite element method in plane Cosserat elasticity. Comput Struct 80:2059-2069

Rovati M, Veber D (2007) Optimal topologies for micropolar solids. Struct Multidiscip Optim 33:47-59

Sharbati E, Naghdabadi R (2006) Computational aspects of the Cosserat finite element analysis of localization phenomena. Comput Mat Science 38:303-315

Sigmund O (1994) Materials with prescribed constitutive parameters: An inverse homogenization problem. Int J Solids Struct 31(17):2313-2329

Sigmund O (1997) On the Design of Compliant Mechanisms Using Topology Optimization. Mech Struct and Mach 25:493524

Sigmund O (2000) A new class of extremal composites. J Mechan $\mathrm{Ph}$ Solids 48:397-428

Sigmund O (2007) Morphology-based black and white filters for topology optimization. Struct Multidiscip Optim 33(4):401424

Sigmund O, Torquato S (1999) Design of smart composite materials using topology optimization. Smart Mater Struct 8:365379

Su W, Liu S (2015) Topology design for maximization of fundamental frequency of couple-stress continuum. Struct Multidiscip Optim 53(3):395-408

Svanberg K (1987) Method of moving asymptotes-a new method for structural optimization. Int $\mathrm{J}$ Numer Methods Eng 24:359-373

Veber D, Taliercio A (2011) Topology optimization of threedimensional non-centrosymmetric micropolar bodies. Struct Multidiscip Optim 45(4):575-587

Wang F, Lazarov BS, Sigmund O (2011) On projection methods, convergence and robust formulations in topology optimization. Struct Mutltidiscip Opt 43(6):767-784

Xia L, Li Z-M, Breitkopf P (2015) Design of materials using topology optimization and energy-based homogenization approach in Matlab. Struct Multidisc Optim 52:1229?1241

Yang W, Li Z-M, Shi W, Xie B-H, Yang M-B (2004) Review On auxetic materials. J of Mater Science 39:3269-3279

Yuan X, Tomita Y (2001) Effective properties of Cosserat composites with periodic microstructure. Mech Res Commun 28(3):265-270 